\title{
RFID: Grundlagen und Potenziale
}

\author{
PROF. DR.-ING. LUDGER OVERMEYER, \\ DiPL.-ING. STEFAN VOGELER \\ UNIVERSITÄT HANNOVER, INSTITUT FÜR TRANSPORT- UND AUTOMATISIERUNGSTECHNIK (ITA)
}

Die Radio Frequenz Identifikation (RFID) erlaubt es, Objekte mit Transpondern zu versehen und sie dadurch eindeutig zu identifizieren sowie drahtlos Daten auszutauschen. Das Potenzial der RFID erstreckt sich dabei weit über die einfache Identifikation von Objekten und bietet die Möglichkeit, Daten lokal zu speichern, sensorisch zu erfassen und am Objekt zu verarbeiten bzw. Steuerungs-entscheidungen zu treffen. Für die volle Ausschöpfung dieses Potenzials sind technologische Fragestellungen der Integration der RFID in technische Systeme zu erforschen, Standardisierungsvorhaben mit dem Ziel einer global einsetzbaren Technik voranzutreiben und Anwendungen zu forcieren, in denen Transponder über die Grenzen geschlossener Kreise eingesetzt werden. Vision ist ein universell einsetzbares, kostengünstiges Processing Label (PL), das die Funktionalitäten der Datenspeicherung, Sensordatenerfassung und Datenverarbeitung vereint.

Radio Frequency Identification (RFID) is a technology for the contactless identification of objects, persons or animals and for the exchange of data using the radio frequency spectrum. The communication is established between a mobile or stationary interrogator and a transponder attached to the object to be identified. As recent transponder comprise rewritable memory, micro controllers or interfaces for sensors RFID can be used beyond identification purposes to store, to process and to generate data locally. In order to benefit from this potential, the integration of RFID into technical systems has to be examined. Furthermore, standardisation issues have to be addressed aiming at worldwide compatibility of RFID systems. Open applications where the transponder is used during different production and use stages of a product should be preferred to closed loop systems. A vision for the near future is a Processing Label (PL) combining the capacity to store data, to process information from sensors and to make local control decisions.

\section{Grundlagen der Radio Frequenz Identifikation}

\subsection{Was ist RFID?}

Sich verändernde Fertigungstiefen innerhalb der Unternehmen, komplexere Produkte und auch der Wandel zur Dienstleistungsgesellschaft stellen immer höhere Anforderungen an die Organisation der Produktion und des Warenflusses, an Logistiksysteme und damit gleichzeitig an automatische Identifikationsverfahren (Auto-ID Verfahren). Seit den 70er Jahren gilt der Barcode als die dominierende Auto-ID-Technologie. Obwohl moderne Barcodescanner hohe Leseraten ermöglichen, verbleiben Probleme bei verschmutzten oder verdeckten Labeln. Daten sind nur in begrenzter Menge speicherbar und nicht aktualisierbar.

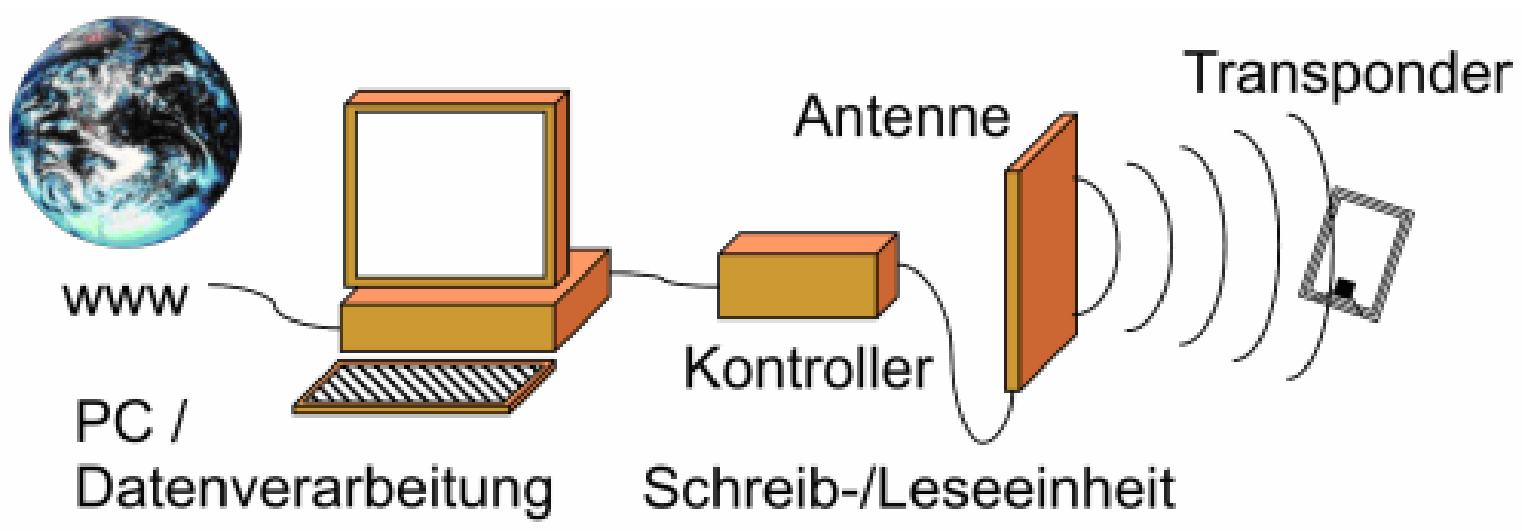

Abbildung 1: RFID-System 
Die Radio Frequenz Identifikation (RFID) stellt dagegen ein Verfahren dar, das es erlaubt, Personen und Objekte eindeutig, schnell und berührungslos zu identifizieren. Ein RFID-System besteht in der Regel aus mindestens einer Schreib-/Leseeinheit, die Daten mit Transpondern - dem Datenmedium der RFID - austauschen kann. Die Schreib-/Leseeinheit umfasst eine Antenne, einen Antennenkontroller und eine Recheneinheit, letztere oft in Form eines PC oder einer Steuerung (vgl. Abb. 1). Eine entsprechend kleinere Antenne mit einem Chip, der als Speicher und Kontroller dient, bildet im einfachsten Fall den Transponder. Der Begriff Transponder setzt sich aus „transmit“ und „respond“ zusammen und beschreibt damit wortwörtlich die Funktion als Antwortsendegerät. Obwohl die Technologie schon seit den 40er Jahren bekannt ist, erfolgte der kommerzielle Durchbruch erst ab 1960 mit sogenannten 1-bit-Transpondern als Diebstahlsicherung in Kaufhäusern. Leistungsfähige Fertigungsverfahren in der Halbleiterindustrie und die gestiegene Nachfrage nach Transpondern haben zu sinkenden Preisen für RFID-Systeme geführt, deren breiten Einsatz neben offenen technologischen und Normungsfragen lange Zeit hauptsächlich die hohen Kosten limitiert haben. RFID wird mittlerweile von der Tieridentifikation über die Behältererkennung, von Zugangssystemen über Werkzeugerkennung bis hin zu Fahrzeugleitsystemen angewendet [Finkenzeller02].

\subsection{Unterscheidungskriterien}

Allen Systemen gemein ist die Datenübertragung zwischen Lesegerät und Transponder über die Luftschnittstelle. Eine Differenzierung der verschiedenen Systeme ist nach unterschiedlichen Kriterien möglich - z.B. nach der Schreib-/ Lesereichweite, der Speichergröße, der Funktionalität, der Energieversorgung oder des Kommunikationsprinzips, etc. Letztlich entscheidet die Betriebsfrequenz über die wesentlichen Eigenschaften des Systems und beeinflusst vorher genannte Kriterien entscheidend.

RFID-Systeme arbeiten in einem Bereich von $100 \mathrm{kHz}$ bis 5,8 GHz, in dem sie sich mehrere Frequenzbänder mit anderen Funkanwendungen - wie z.B. Behördenfunk, Radio oder Betriebsfunk - teilen (vgl. Abb. 2). Die Zulassung für die entsprechenden Frequenzen regeln nationale und internationale Fernmeldevorschriften. Im Wesentlichen lassen sich vier charakteristische Frequenzbereiche unterscheiden. Low Frequency (LF) Systeme nutzen Frequenzen zwischen 100-135 kHz und werden typischerweise bei der Tieridentifikation eingesetzt. High Frequency (HF) bezeichnet das Band um 13,56 MHz. Im Zuge des Booms des elektronischen Produktkodes (EPC) und der damit verbundenen Anforderungen an günstige, über größere Reichweiten lesbare Transponder erlebt der Ultra High Frequency (UHF) Bereich um $868 \mathrm{MHz}$ in Europa und $915 \mathrm{MHz}$ in den USA einen deutlichen Aufschwung. Micro Wave (MW) Systeme um 2,45 GHz und höher sind meist nur als aktive Funksysteme im Einsatz.

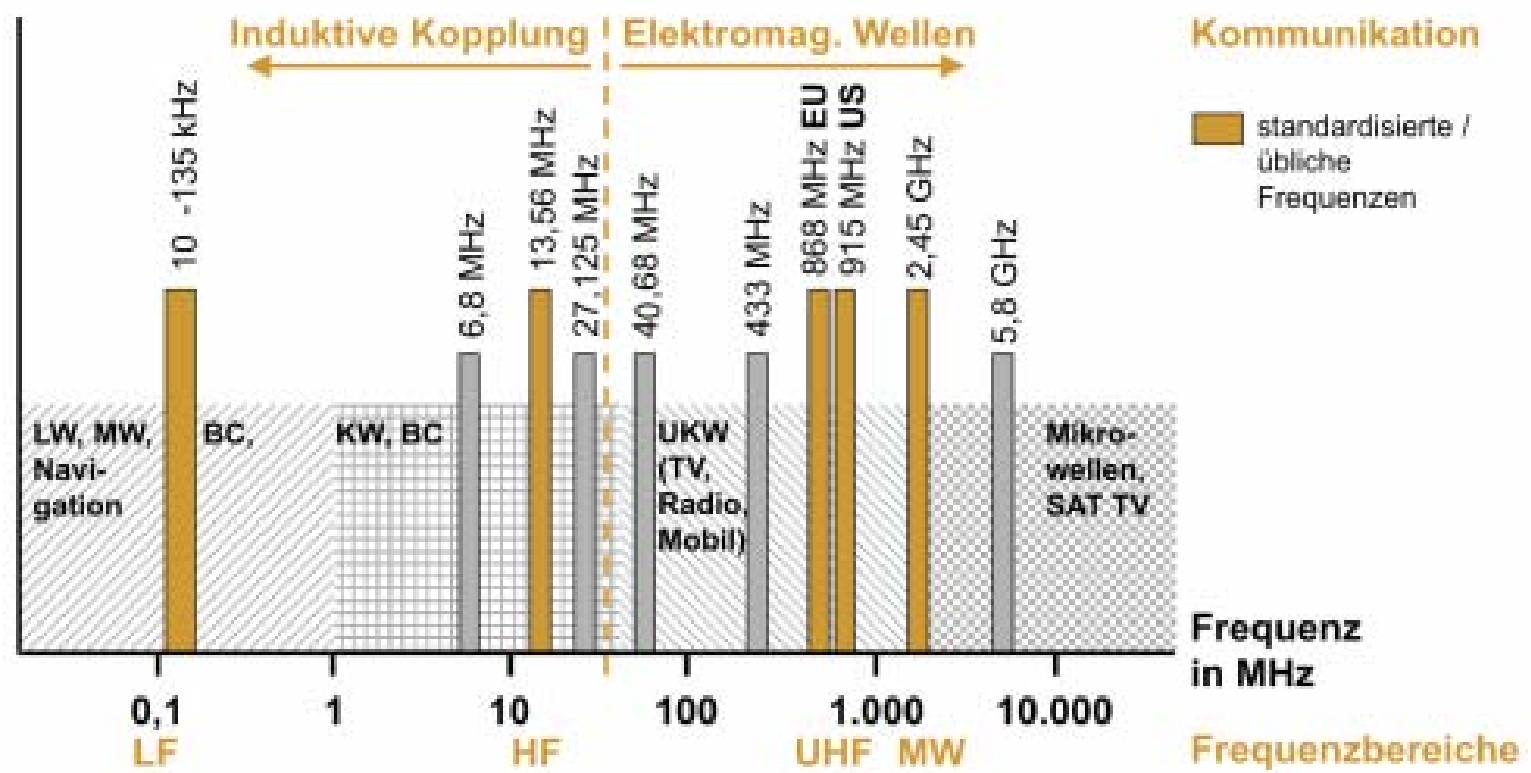

Abbildung 2: RFID Betriebsfrequenzen (nach [Finkenzeller02], [Infinion03])

Die kurzen Anmerkungen zu Besonderheiten und Anwendung bei der Auflistung der vier Hauptfrequenzbereiche verdeutlichen bereits den Einfluss der Frequenzen auf die Systemeigenschaften. Innerhalb eines bestimmten Abstandes um die Schreibleseeinheit können Transponder induktiv gekoppelt betrieben werden (vgl. Abb. 2), solange der Abstand kleiner als der Quotient aus der Wellenlänge und $2 \pi$ ist. In diesem Modus lässt sich der Transponder relativ gut aus dem Feld des Schreib-/ Lesegerätes mit Energie speisen und kommt so ohne eigene Leistungsversorgung aus (passives System). 
Die Kommunikation erfolgt durch Modifikation des Transponder-antennenschwingkreises und somit der Gegeninduktivität, mit der der Tag das Feld des Schreib-/Lesegerätes beeinflusst. Nach der oben erläuterten Beziehung erreichen induktive Verfahren bei Frequenzen über $30 \mathrm{MHz}$ (UHF- und MW-Systeme) nur noch minimale theoretische Reichweiten. Bei höherfrequenten Systemen beruht die Kommunikation daher auf dem Spiegeln (Back-Scatter) bzw. dem aktiven Senden (Funkprinzip) von elektromagnetischen Wellen. Mit der so erreichten höheren Reichweite wird die passive Energieversorgung des Tags schwieriger. UHF-Transponder liegen folglich entweder nur als Read Only (R/O) bzw. mit kleinem Nutzspeicher vor oder werden als aktive Systeme mit eigener Leistungsversorgung angeboten.

Aktive Systeme nutzen die Energiequelle sowohl zur Kommunikation als auch zum Betrieb des Chips selbst. Semi-aktive Systeme als Mischformen aus aktiven und passiven Systemen stützen sich entweder zur Kommunikation - z.B. mit dem Ziel hoher Reichweiten - oder zum Betrieb des Chips - z.B. bei zusätzlicher Sensorik - auf die mitgeführte Spannungsquelle.

Die Komplexität von Transpondern reicht von der 1-bit Diebstahlsicherung bis zu Systemen mit 128 kByte, von R/O bis zu Tags mit wiederbeschreibbarem Speicher, Mikrokontroller und Sensorschnittstelle.

Nach der Reichweite lassen sich vier Gruppen von RFID-Systemen gemäß Abb. 3 unterscheiden. Wie eingangs erwähnt, ist die maximale Schreib-/Lesereichweite neben der Transpondergröße und Leistungsparametern der Schreib-/ Leseeinheit von der Betriebsfrequenz abhängig. Für sicherheitsrelevante Anwendungen werden zum Schutz vor Datenmissbrauch häufig extrem kurze Reichweiten (Close Coupling) gefordert.

\section{System:}

\section{Close coupling}

\section{Remote coupling}

Long range

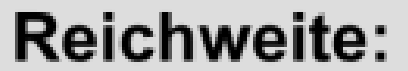

$1 \mathrm{~cm}$

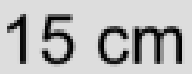

$100 \mathrm{~cm}$

$$
>1 \mathrm{~m}
$$

(bis $300 \mathrm{~m}$ )
Beispiel:

Bankkarten

Zugangskontrollen

Logistikanwendungen

Fahrzeugkennzeichnung

Abbildung 3: Reichweiten von RFID-Systemen

In Abbildung 4 sind alle gängigen Differenzierungskriterien für Transponder dargestellt. Die Abhängigkeit der Kriterien untereinander wird schnell am Beispiel eines passiven $868 \mathrm{MHz}$ Transponders deutlich. Zur Kommunikation mit vernünftigen Reichweiten kommt nur Back-Scatter in Frage, wodurch Reichweiten deutlich über einen Meter - also Long Range - erreicht werden. Die weiter oben erläuterten Schwierigkeiten der Leistungsübertragung lassen mit R/O oder Read Write (R/W) Chips mit kleinen Speicherbereichen nur wenig komplexe Systeme zu. 


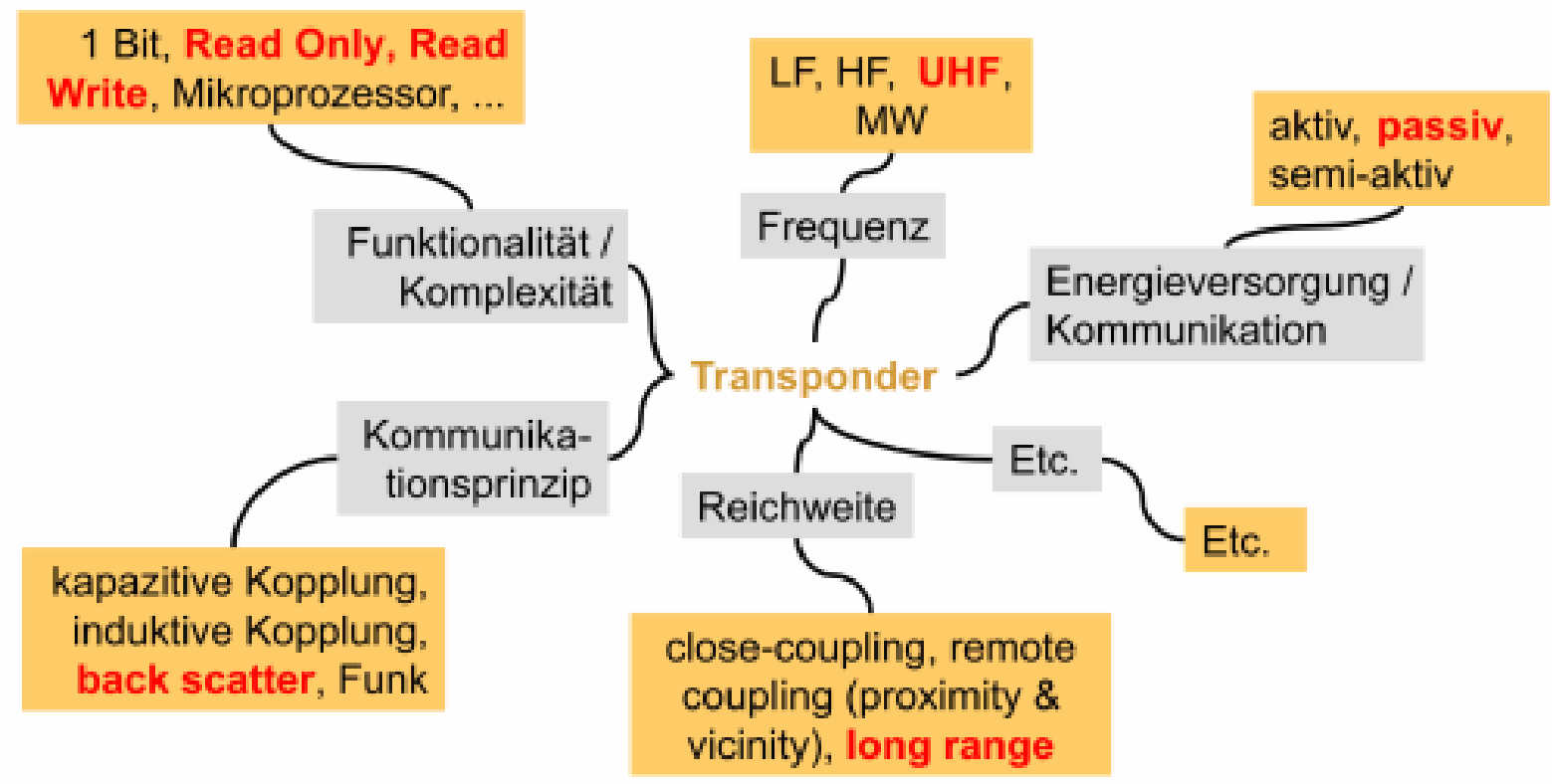

\section{Beispiele für Verknüpfung der Kriterien: passiver $868 \mathrm{MHz}$ Transponder}

\section{Abbildung 4: Einteilungskriterien}

Der Erfolg der RFID ist eng mit der Existenz praktikabler Standards und Normen verbunden, die eine länderübergreifende Systemkompabilität gewährleisten. Regelungen, die RFID-Anwendungen betreffen, fallen zum einen in den Bereich nationaler und internationaler Regulierungsbehörden für Fernmeldetechnik (ETSI, CEPT, etc. sind diese Einrichtungen allgemein unter ihren Abkürzungen bekannt, oder sollten sie aufgelöst werden?), zum anderen beschäftigen sich technische Gremien wie ISO / IEC oder EPCglobal mit der Definition von Hard- und Softwarestandards. Besonders für den Hochfrequenzbereich ist mit den Normen ISO 14443 für Proximity Cards, ISO 15693 für Vicinity Cards und ISO 18000-3 für die Luftschnittstelle eine gute Standardisierung vorhanden.

Die 2003 von EAN International und dem Uniform Code Council gegründete EPCglobal, Inc. entwickelt technische und wirtschaftliche Standards für das Electronic Product Code (EPC)-Netzwerk. Über den EPC (s.o., schon aufgelöst) sollen Waren in der Versorgungskette eineindeutig mittels aufgebrachter Transponder gekennzeichnet werden können.

\section{Potenziale und Anwendungen in der Forschung}

\subsection{Speichern, Messen, Rechnen}

Der Breite an vorhandenen Systemen stehen mindestens ebenso zahlreiche Anwendungen gegenüber. Abbildung 5 bietet eine Übersicht gängiger Anwendungsbeispiele für die RFID in unterschiedlichsten Bereichen. Die meisten vorhandenen Systeme sind sogenannte closed-loop Lösungen, also geschlossene Kreisläufe z.B. in der Automobilmontage. Für ein günstiges Kosten/Nutzen-Verhältnis der RFID ist der Einsatz über Unternehmensgrenzen hinweg ein angestrebtes Ziel, das im Wesentlichen von erwarteten Kostenreduzierungen im Bereich der Hardware und von den Erfolgen voranschreitender Standardisierungsbemühungen abhängig ist [Booz04]. 


\begin{tabular}{|c|c|c|c|c|c|}
\hline $\begin{array}{l}\text { Verleih- } \\
\text { systeme }\end{array}$ & $\begin{array}{l}\text { Wäscherei, } \\
\text { Werkzeug, } \\
\text { Dienstkleidung. } \\
\text { Sportartikel, } \\
\text { Bibliotheken }\end{array}$ & Behälter ID & $\begin{array}{l}\text { Gasflaschen, } \\
\text { Chemikalienbehalter, } \\
\text { Abfallmanagement }\end{array}$ & $\begin{array}{l}\text { Bargeldloses } \\
\text { Bezahlen, } \\
\text { Treue- } \\
\text { programme }\end{array}$ & $\begin{array}{l}\text { Kreditkarten, } \\
\text { Kundenkarten } \\
\text { (CRM) }\end{array}$ \\
\hline $\begin{array}{l}\text { Echtheits- } \\
\text { zertifikat }\end{array}$ & $\begin{array}{l}\text { Casinochips, } \\
\text { Ausweise, Vertrăge, } \\
\text { Urkunden, Luxusgûter }\end{array}$ & Sport & $\begin{array}{l}\text { Zeitnehmer, } \\
\text { Veranstaltungs- } \\
\text { management }\end{array}$ & $\begin{array}{l}\text { Medizin- } \\
\text { technik / } \\
\text { Labor }\end{array}$ & $\begin{array}{l}\text { Bestand, Diagnose, } \\
\text { Therapie, Endogene } \\
\text { Sensoren }\end{array}$ \\
\hline Logistik & $\begin{array}{l}\text { Lagersysteme, Supply } \\
\text { Chain Management, } \\
\text { Kühlketten, } \\
\text { Paketdienst, } \\
\text { Fluggepäck }\end{array}$ & $\begin{array}{l}\text { Industrie- } \\
\text { automation }\end{array}$ & $\begin{array}{l}\text { Werkzeug ID, } \\
\text { Prozesssteuerung. } \\
\text { Prozessüber- } \\
\text { wachung, Sensorik }\end{array}$ & $\begin{array}{l}\text { Verkehrs- } \\
\text { systeme }\end{array}$ & $\begin{array}{l}\text { Euro-Baseline, } \\
\text { Container ID, Maut, } \\
\text { digitales Nummem- } \\
\text { schild, Flotten- } \\
\text { management, } \\
\text { autonomes Fahren }\end{array}$ \\
\hline Ticketing & OPNV, Lufthansa, Ski & Tier ID & $\begin{array}{l}\text { Tierzucht, } \\
\text { Brieftauben, Haustiere }\end{array}$ & Automobil & $\begin{array}{l}\text { Wegfahrsperre, } \\
\text { Reifendrucksensor }\end{array}$ \\
\hline $\begin{array}{l}\text { Zutritts- } \\
\text { kontrolle }\end{array}$ & $\begin{array}{l}\text { Industrie, Behörden, } \\
\text { Events }\end{array}$ & $\begin{array}{l}\text { Diebstahl- } \\
\text { sicherung }\end{array}$ & $\begin{array}{l}\text { Electronic Article } \\
\text { Surveillance (EAS) }\end{array}$ & Tourismus & $\begin{array}{l}\text { Zugang, Rabatte, } \\
\text { Zahlsysteme }\end{array}$ \\
\hline
\end{tabular}

Abbildung 5: Typische RFID-Anwendungen

Die Forschung am Institut für Transport- und Automatisierungstechnik (ITA) zielt auf intelligente Anwendungen insbesondere für passive RFID-Systeme, die Anpassung der Transponder an die jeweilige Systemumwelt und die benötigten leistungsfähigen Verfahren der Verbindungstechnik. Vision der Forschungsarbeiten sind kostengünstige Processing Label (PL), die die Funktionen der Datenspeicherung, der Kommunikation mit der Umwelt durch Datenaustausch und Messdatenerfassung sowie einer lokal verfügbaren Rechenleistung vereinen. Die drei genannten Teilfunktionen werden im Rahmen unterschiedlicher Projekte erforscht. Ein Fokus liegt hierbei stets auf der Ermittlung bislang unbekannter Anwendungsfelder, der Nutzung einer erweiterten Technologie bei Standardanwendungen und der Integration der Transponder in technische Systeme.

\subsection{Speichern: Verbindung von Objekt und Information}

Transponder als Datenspeicher realisieren eine Verbindung von Objekt und objektbezogener Information und umgehen die Nachteile von Medienbrüchen, wie z.B. Datenverluste oder Fehldaten als Folge manueller Dateneingabe. Durch die lokale Datenspeicherung werden einerseits zentrale Datenbanken entlastet, die bei anderen Autoidentifikationstechniken einem Objekt objektbezogene Daten über eine Kennung zuweisen, andererseits lassen sich Anwendungen schneller realisieren, weil nicht sofort eine netzgebundene Datenbank zur Verfügung stehen muss [Booz04], [Finkenzeller02].

\subsection{Speichern: Gepäckverfolgung am Flughafen}

Seit den Geschehnissen des 11. Septembers 2001 fordert der Gesetzgeber insbesondere in den Vereinigten Staaten von Amerika eine lückenlose Nachverfolgbarkeit von Fluggepäck. Neben dem Sicherheitsrisiko ausgehend von nicht eindeutig identifizierten Gepäckstücken besteht bei den Fluggesellschaften ein erhebliches Einspar-potential, wenn der Verlust von Gepäck vermieden bzw. verlorenes Gepäck sicher seinem Eigentümer zugeordnet werden kann. Zahlreiche Flughäfen und Fluggesellschaften experimentieren daher mit Smart-Labeln zur Gepäckkennzeichnung [OConor04].

Am ITA wird der Einsatz beschreibbarer Transponder im Rahmen der Gepäckverfolgung erforscht. Indem die Resultate der Röntgenuntersuchungen direkt im Smart Label und somit am Gepäckstück gespeichert werden, realisiert man mit der Beschreibbarkeit einen zusätzlichen Sicherheitsgewinn (vgl. Abb. 6). Das Vertauschen indizierter Gepäckstücke in der Fördertechnik ist damit nahezu ausgeschlossen. Neben Sicherheitsaspekten ergeben sich potenzielle Einsparungen im Bereich der Fördertechnik durch Reduktion der Anzahl redundanter Ausschleusstrecken. 


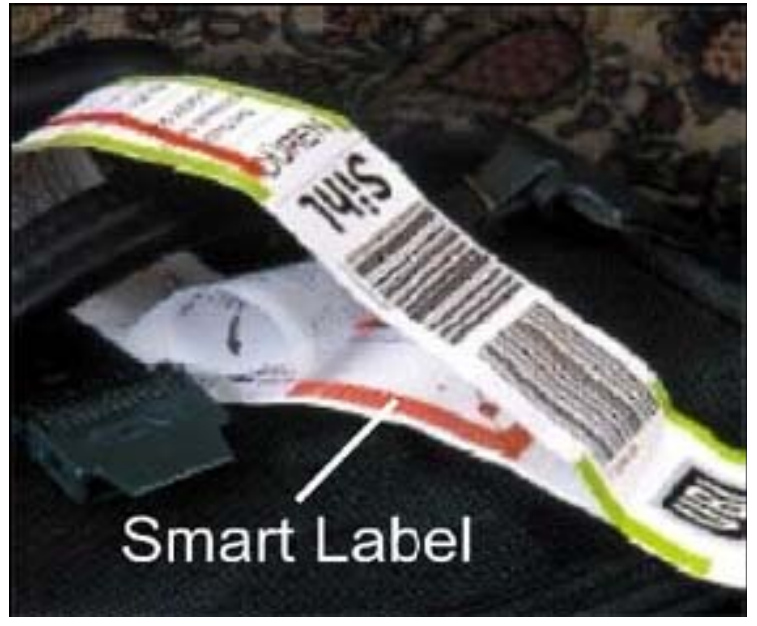

Abbildung 6: Gepäckidentifikation

\subsection{Speichern: Transpondergestütztes Fahrzeugleitsystem}

Stand der Technik für autonome Fahrzeugsysteme im Routenwerksverkehr sind induktive Leitdrahtführungen oder einfache niederfrequente RFID-Systeme. Im Fall einer Streckenänderung gestaltet sich das Aufbringen neuer Transponder zwar weniger aufwändig als das Verlegen neuer Leitdrähte, aber auch beim Einsatz einfacher Transponder können dem Fahrzeug keine Vorabinformationen über den weiteren Streckenverlauf übermittelt werden.

In einem aktuellen Projekt wird erforscht, wie HF-RFID-Systeme zur Fahrzeug-steuerung eingesetzt werden können. Durch die höheren Übertragungsraten der HF-Systeme sind höhere Fahrgeschwin-digkeiten realisierbar. Außerdem können mehr Daten vom Chip ausgelesen und somit Orts- und Streckeninformationen an das Fahrzeug übertragen werden. Das Fahrzeug kennt so nicht nur seine genaue Position, sondern verfügt zusätzlich über Informationen, mit welcher Geschwindigkeit es in welcher Richtung weiterzufahren hat. Der Transponder fungiert damit als virtuelles Verkehrsschild, das durch die Wiederbeschreibbarkeit des Speicherchips auch zeitlich veränderliche Ereignisse - wie Bauvorhaben - berücksichtigen kann. Ist ein in Raster eingeteiltes Gelände mit Transpondern ausgestattet, lassen sich Fahrrouten beliebig verändern.

Langfristig kann die Transpondertechnologie in Kombination mit Daten des Global Postioning Systems (GPS) sowie den Sensorinformationen der Fahrassistenzsysteme autonomes Fahren auch unter realen Verkehrsbedingungen ermöglichen.

Hauptansatzpunkt für die Forschung ist neben der Definition geeigneter Datenstrukturen die Integration der Transponder in Fahrbahnoberflächen (vgl. Abb. 7). Der Tag muss sowohl den Belastungen standhalten, die auf eine Fahrbahnoberfläche einwirken, als auch einfach und kostengünstig aufbringbar sein.

\section{Lackabdeckung}

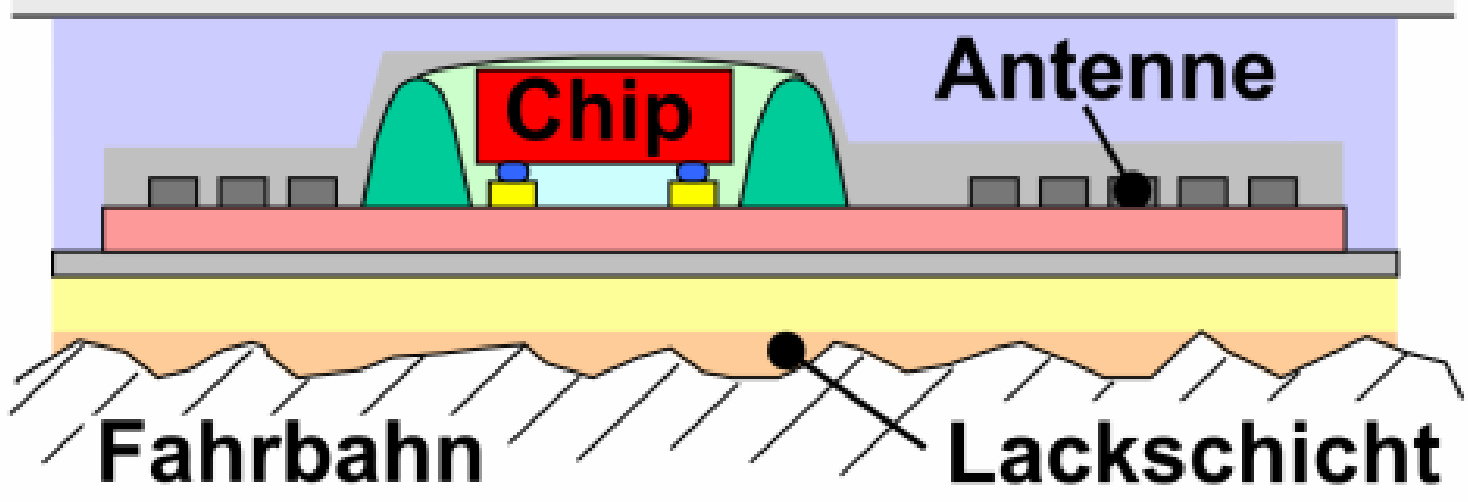

Abbildung 7: Transponder auf der Fahrbahn 
Ähnliche Systeme sind allgemein zur Navigation denkbar und können für andere Verkehrsteilnehmer nutzbar gemacht werden. Denkbar ist der transpondergestützte Stadtführer oder eine Orientierungshilfe für sehbehinderte Menschen [Texas04].

\subsection{Speichern: Transponder in Werkzeugmaschinen}

Kleinere Losgrößen und komplexe Bauteile erfordern anpassungsfähige Werkzeugmaschinen. Bei der Ersteinrichtung bzw. Rekonfiguration einer Werkzeugmaschine nimmt die Programmierung der Steuerung viel Zeit in Anspruch. Eine plug\&play-ähnliche Funktionalität, die es der Maschine erlaubt, vorhandene Baugruppen sicher zu erkennen, spart Zeit bei der Maschineneinrichtung und vermeidet Fehler bei der Konfiguration.

Da Werkzeugmaschinen in den seltensten Fällen echte Serienprodukte sind, ist die Erstellung einer Maschinendokumentation mit erheblichem Aufwand verbunden. Lassen sich die Komponenten beim Zusammenbau einfach in ein EDV-System einlesen, besteht die Möglichkeit der automatischen Generierung vollständiger und richtiger Maschinenbeschreibungen.

Nach der Inbetriebnahme werden die Maschinen oft unterschiedlich stark beansprucht, so dass eine Bewertung des Zeitwertes einzelner Maschinen-baugruppen, die bedarfsgerechte Instandhaltungsplanung und Klärung von Gewährleistungsfragen schwer fällt.

Durch die Erforschung des Einsatzes drahtloser Kommunikationssysteme in den Komponenten von Werkzeugmaschinen soll den oben erläuterten Problemen entgegengewirkt werden. Erste Ansätze zum Einsatz solcher Systeme sind vorrangig im Bereich der Werkzeugidentifikation bekannt [Finkenzeller02]. Probleme entstehen durch das metallische Umfeld der Maschine, das die drahtlose Kommunikation je nach Frequenz durch Wirbelstromeinflüsse oder unkontrollierte Reflektionen stört.

\section{8. $\quad$ Messen - Datenerfassung am Objekt}

Neben der bisherigen Nutzung der Transponder zur Datenspeicherung ergibt sich durch die Ankopplung von Sensoren ein weiteres Einsatzgebiet [Overmeyer03]. Besonders die drahtlose Messdatenübermittlung macht Messtransponder für die Anwendung in bewegten Systemen interessant.

\subsection{Messen: Transponder im Fördergurt - Schlitzschutz}

Gurtförderanlagen transportieren Schüttgut nicht selten über mehrere Kilometer und teilweise unter schwierigen Umgebungsbedingungen. Die Anlagen stellen für den Betreiber ein bedeutendes Investitionsvolumen dar, wobei 40\% der Anlagenkosten auf den Gurt selbst entfallen.

Durch einen Riss im Fördergurt geht das geförderte Gut verloren und der defekte Gurt kann die Anlage selbst massiv beschädigen. Betreiber von Gurtanlagen lassen sich daher von den Gurtherstellern die Haltbarkeit des Gurtes garantieren. Zur Minimierung des Risikos von Gurtrissen und der Eingrenzung deren Folgen werden Schlitzsensoren eingesetzt [Keller04]; [Alles01].

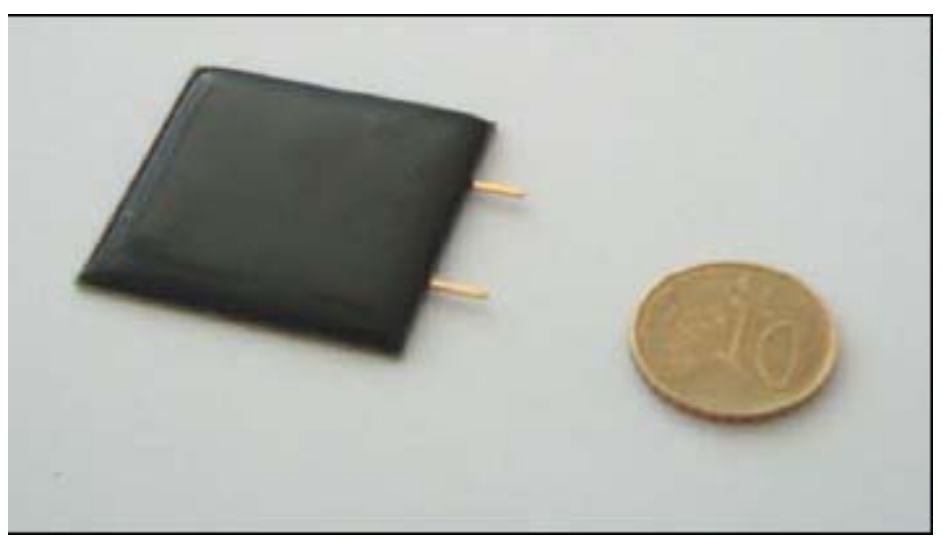

Abbildung 8: Schlitzschutztransponder

Existierende Systeme bestehen aus Transpondern, Induktionsspulen zur Rissdetektion und zwei versetzt angeordneten Leseantennen [Keller04]. Diese Vorgehensweise ist mit einer Reihe von Problemen behaftet. Neben dem Bedarf an zwei Antennen stört die aufwändige Integration von Spule und Transponder sowie deren Ausrichtung zueinander. Die Kommunikation über Induktion in Spule und Transponder resultiert in Schwierigkeiten beim Lesen der Signale.

Forschungsgegenstand sind daher Transponder mit Sensoreingang, die für eine direkte Rissdetektion die Durchgängikeit der Messspule über Sensoreingänge erfassen. Sensortransponder und Spule werden in den Gurt integriert und mit nur einer Antenne ausgelesen. 
Unterbricht ein Gurtriss die Spule, wird dies über den Sensoreingang detektiert und die Havarie vom Transponder über eine 1bit-wertige Änderung in der Identifikationsnummer gemeldet. Durch den Betrieb im Hochfrequenzbereich werden höhere Gurtumlaufgeschwindigkeiten sowie eine zuverlässigere Datenübertragung über größere Distanzen erreicht.

Neben der Sensorfunktion leisten eingebettete Transponder einen wichtigen Beitrag zur Betriebsdatenerfassung und Instandhaltungsplanung. Die RFID-Technik ersetzt optische Markierungen von Gurtsystemen, die durch Verschleiß schnell unkenntlich werden und ermöglicht so das Positionieren von Gurtabschnitten zur Inspektion [Dietz01].

\subsection{Messen: Messende Transponder in fördertechnischen Anlagen}

Transponder mit Druck- und Temperatursensoren sind bereits am Markt erhältlich. Ein dehnungsmessender Transponder wird derzeit am ITA entwickelt. Neben der Realisierung der Sensorfunktion stellen die physikalischen Anforderungen an einen Transponder im Förderband die größten Herausforderungen dar. Einerseits muss das System Druck und Temperatur während der Einlaminierung tolerieren, andererseits den Biegewechselfestigkeitsanforderungen genügen, die an Transportbänder im Betrieb gestellt werden [Overmeyer03].

Bei der Implementierung auftretende Temperaturen bis $170^{\circ} \mathrm{C}$ und Drücke bis 7 bar führten bei keinem der getesteten Transponder zu einem Funktionsausfall. Um den Betrieb zu simulieren, werden die Transportbänder einer Biegewechselprüfung unterzogen, bei der die implementierten Transponder sowohl einer Zug- als auch einer Biegebeanspruchung ausgesetzt werden. Die Biegefestigkeit der für erste Tests verwendeten Smart Label lässt sich durch verstärkte Foliensubstrate und spezielle Einbettungsmaßnahmen um den Faktor 2 steigern.

Weitere Versuche wurden mit biegesteifen Transpondern durchgeführt. Um Spannungsrisse zu vermeiden, darf hierbei die Kantenlänge des Transponders 9 mm nicht überschreiten. Als geeignetes Transponder-Trägermaterial hat sich ein Verbundwerkstoff aus Glasfaser und Epoxydharz erwiesen. Da auf dem Markt Transponder mit zuvor beschriebenen Eigenschaften standardmäßig nicht erhältlich sind, werden die Untersuchungen derzeit mit am ITA hergestellten Labormustern erfolgreich durchgeführt.

Die eigentlichen Versuche erfolgen mit einem speziell entwickelten Messtransponder. Statt auf das traditionelle Wire-Bond-Verfahren zur Integration des Transponderchips auf dem Substrat zurückzugreifen, wurde im Interesse einer haltbareren flexiblen Verbindung die Kontaktierung des Chips mittels leitfähigen Klebers realisiert. Neben dem Schutz der Chipverbindung wird am Verguss der Messelektronik mit verschiedenen Werkstoffen geforscht, um die Bauteile vor mechanischer Beschädigung zu schützen [Wennekamp04]. Zur Dehnungsmessung werden zu einer Vollbrücke verschaltete Dehnungsmessstreifen (DMS) eingesetzt. Der messende Teil des Transponders wurde bei den ersten Versuchen mit der Gewebeschicht des Transportbandes verklebt.

Die Dehnungsraten der Transportbänder überschreiten die Grenzen des Dauerfestigkeitsbereichs der DMS. Für weitere Versuche ist daher der Einsatz eines dehnungsübersetzenden Messaufnehmers vorzusehen. Derzeit werden verschiedene Varianten in Versuchsreihen erprobt.

Auf längere Sicht ist die Intergration der Messelektronik und Transponderfunktion in einem Chip anzustreben.

\subsection{Rechnen - lokale Datenverarbeitung}

Transponder mit eigenem Mikrokontroller sind analog zu Smart Cards in der Lage, einfache Programme abzuarbeiten und so Daten lokal zu verarbeiten und Entscheidungen herbeizuführen.

\subsection{Rechnen: Logistikdatenerfassung und -verarbeitung in der Produktion}

Die Produktion der Zukunft muss flexibel auf sich ändernde Rahmenbedingungen reagieren können. Eine wesentliche Voraussetzung zur Umsetzung flexibler dezentraler Fertigungslinien ist ein leistungsfähiges SupplyChain-Management.

Die Erfassung der erforderlichen Betriebsdaten zur Steuerung und Optimierung von Produktionsabläufen erfolgt größtenteils noch manuell. Kostenintensive Personalbindung, Fehleranfälligkeit durch Medienbrüche und zeitlich versetzte Erfassung von Daten machen dieses Vorgehen für die Produktionssteuerung nur bedingt einsetzbar.

Um die genannten Probleme bei der Datenerfassung zu lösen und eine dezentrale Datenspeicherung und Verarbeitung zu ermöglichen, wird am ITA der Einsatz der RFID-Technolgie mit dem Ziel der Entwicklung eines Pre-Processing Labels (PPL) erforscht.

Erste Einsätze der RFID-Technik zur Produktionssteuerung sind aus der Automobilindustrie bekannt, wo die papierbasierten Bauzettel stellenweise durch Transponder ersetzt wurden [Schröder02]. Mit den Fortschritten der letzten Jahre im Bereich Smart Label wird der breite Einsatz in der Produktion möglich. 
Mit gängigen Smart Labeln können Produkte echtzeitnah und berührungslos eindeutig identifiziert werden, wesentliche Produktdaten am Produkt gespeichert und verändert bzw. ergänzt werden. Auf die Rechenleistung einer kontaktbehafteten Smart Card mit Mikroprozessor musste bislang verzichtet werden. Eine Kombination der Flexibilität des Smart Label und der Rechenleistung einer Smart Card stellt das PPL dar. Aufgrund des hohen Standardisierungsgrades wurde die Arbeitsfrequenz 13,56 MHz für die Realisierung des PPLs gewählt. Ein Infineon Prozessor stellt mit einem nichtflüchtigen Speicher die Grundlage zur Abarbeitung einfacher Programme und Speicherung von Daten bzw. Programmergebnissen bereit.

Nach der Definition des Chips wurde eine Antennenspule aus silberummanteltem Kupferdraht entwickelt, um eine möglichst hohe Güte des Schwingkreises zu erreichen und damit die Energieversorgung des Chips aus dem Feld zu gewährleisten.

Erste Versuche mit den PPL beschränken sich zunächst auf die Produktionskette innerhalb eines Unternehmens, indem der Datenaustausch bei der Materialbereitstellung mit dem Übertragen der Plandaten auf das PPL beginnt und mit dem Ende der Fertigung abgeschlossen ist. In naher Zukunft ist die Ausdehnung der Anwendung auf die gesamte Wertschöpfungskette geplant (vgl. Abb. 9).

Das PPL entlastet durch seine Rechenkapazität die zentrale Produktionssteuerung und beinhaltet ständig aktuelle Soll- und Istdaten. Eine Reaktion auf Änderungen im Produktionsprozess ist somit ohne größere Umstände oder den Verlust von Daten möglich [Höhn04].

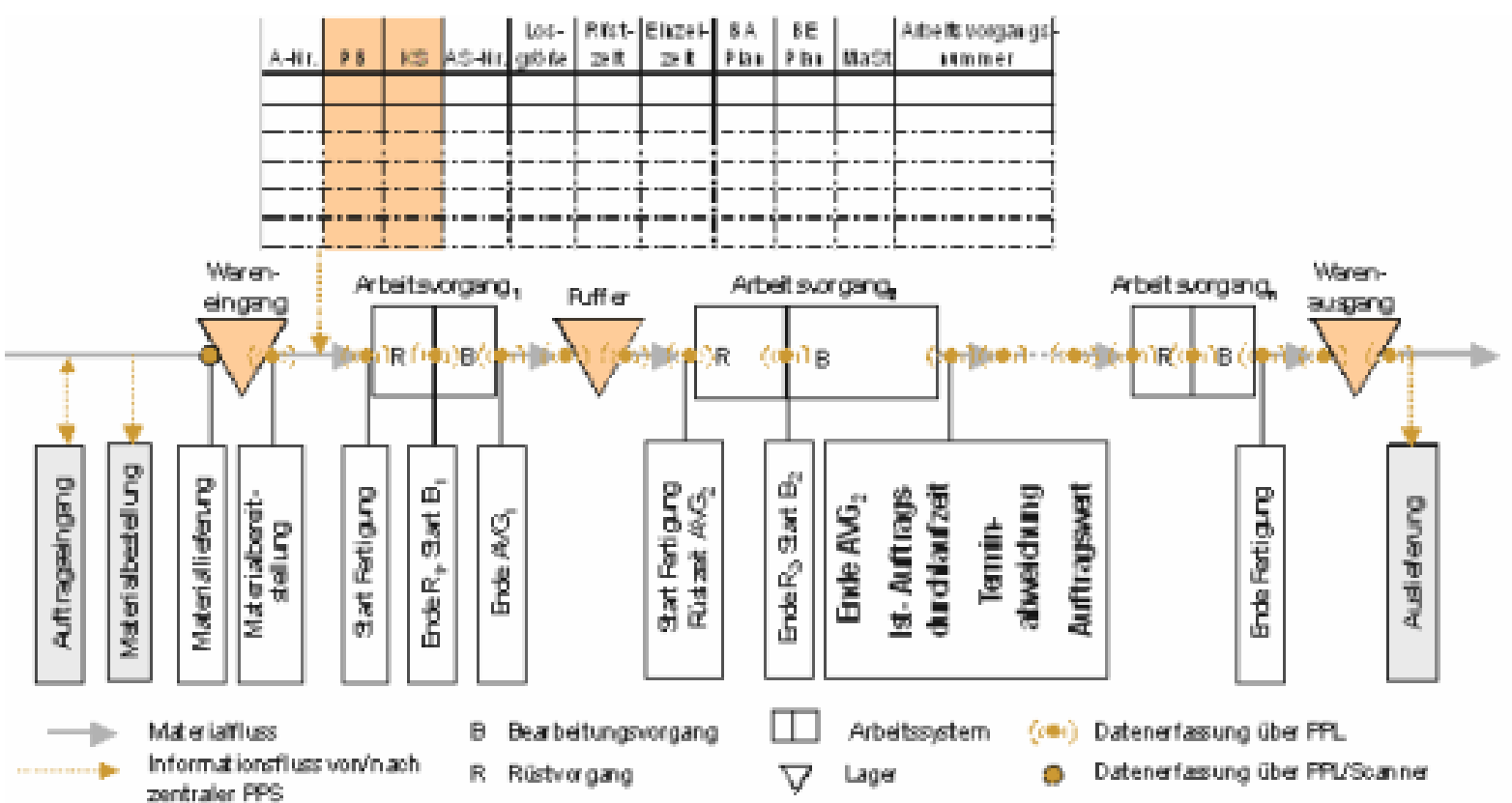

Abbildung 9: Beim Verlassen einer Arbeitsstation überträgt das PPL die berechneten Daten an die Produktionssteuerung

\section{Visionen - die Zukunft der RFID}

Die vergleichsweise hohen Preise für Transponder und mangelnde Standardisierung galten lange Zeit als Haupthindernisse für eine breite Anwendung der drahtlosen Identifikationstechnik. Mittlerweile sind erste Normen verabschiedet und EPC bzw. ISO treiben eine weitere Standardisierung der Technik mit dem Ziel der Schaffung einer globalen Basistechnologieplattform voran. Letztere wird aufgrund steigenden Vertrauens in die Beständigkeit der RFID in höherer Nachfrage und damit sinkenden Transponderpreisen resultieren. 
Preisentwicklung passiver Label

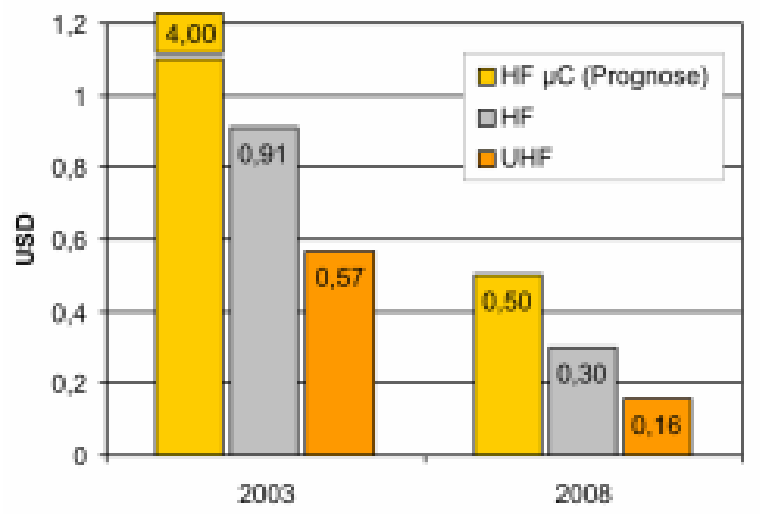

Marktenetwicklung für RFID-Anwendungen

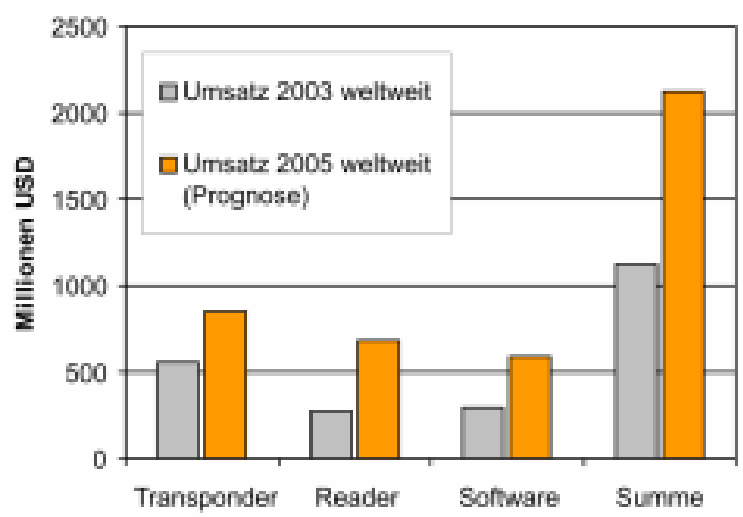

Abbildung 10: Marktdaten (nach [Ward04], [VDC04])

Abbildung 10 verdeutlicht die erwartete Preisentwicklung für passive Label, die bis 2008 im Durchschnitt wahrscheinlich noch nicht die geforderte 5 Eurocent - Marke unterschreiten werden, ihr Preis aber in den nächsten drei Jahren um mehr als 2/3 sinken wird. Auch Marktentwicklungsdaten für die RFID-Anwendungen unterstreichen im Allgemeinen den Wachstumstrend. Erste Systeme sind zwar meist als Closed-LoopAnwendungen konfiguriert, die Entwicklung des Marktes deutet jedoch darauf hin, dass zukünftig verstärkt offene Systeme über Unternehmensgrenzen hinweg eingesetzt werden. Wie sich bereits beim Global System of Mobile Communication (GSM) gezeigt hat, steigt der Nutzen einer neuen Kommunikations- und Identifikationstechnologie oftmals exponentiell mit der Anzahl der Anwendungen an [Booz04).

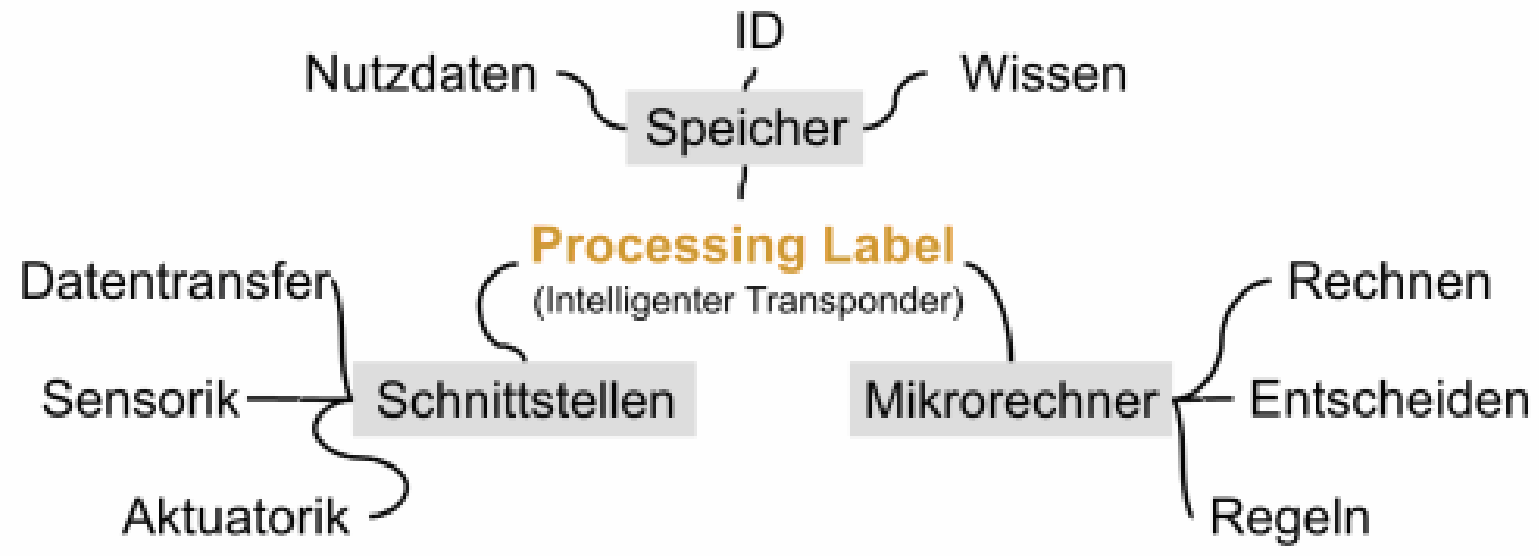

Abbildung 11: Processing Label

Die Erforschung neuartiger RFID-Anwendugen ist ein Schwerpunkt der Tätigkeit am ITA. Die Zahl der Anwendungen und damit sowohl der Nutzen als auch das Kosten-Nutzen-Verhältnis lässt sich durch zwei maßgebliche Strategien realisieren.

Eine Vision für die nahe Zukunft ist die Realisierung des Processing Labels (kommt bereits im Abstract und auf Seite sechs vor), d.h. eines Transponders, der bei passiver Leistungsversorgung die Merkmale Datenspeicherung, Kommunikation und Datenerfassung sowie Rechenleistung in Form eines Mikrocontrollers vereint (vgl. Abb. 11). Das PL versieht vormals einfache Produkte mit Intelligenz und versetzt sie in die Lage, mit ihrer Umwelt zu kommunizieren und dezentralisiert die Speicherung, Erfassung und Verarbeitung von Daten mit dem Ziel, Mensch und Maschine bei der Entscheidungsfindung zu unterstützen, kurze Regelkreise zu erreichen und Objekt und Information untrennbar zu verbinden.

Die Anwendung eines PL umfasst im einfachsten Fall die eindeutige Identifikation und erstreckt sich bis zum Auslösen einer Steuerungsentscheidung als Folge eines bestimmten über die integrierte Sensorik ermittelten Zustandes. Die Umsetzbarkeit des PLs wird mit den Arbeiten zur Einsetzbarkeit des PPLs zur Betriebsdatenerfassung und Produktionssteuerung bereits untersucht. Neben industriellen oder logistischen Anwendungen ist aber auch die Interaktion mit Unterhaltungs- und Haushaltselektronik als Erweiterung bestehender Near-Field-Communication-Ansätze denkbar. 
Der zweite Strategieansatz fordert von künftigen RFID-Anwendungen, den „closed-loop“ innerhalb der Unternehmens- bzw. Abteilungsgrenzen und geschlossener Anwendungsszenarien zu durchbrechen und den Tag über den ganzen Lebenszyklus des mit ihm assoziierten Produkts zu nutzen (vgl. Abb. 12). Ähnlich einer DNA beschreibt der Transponder seinen Träger eineindeutig im Sinne der Identifikation und kann Informationen über wichtige Eigenschaften mitführen, die durch Wiederbeschreibbarkeit immer dem aktuellen Stand entsprechen.

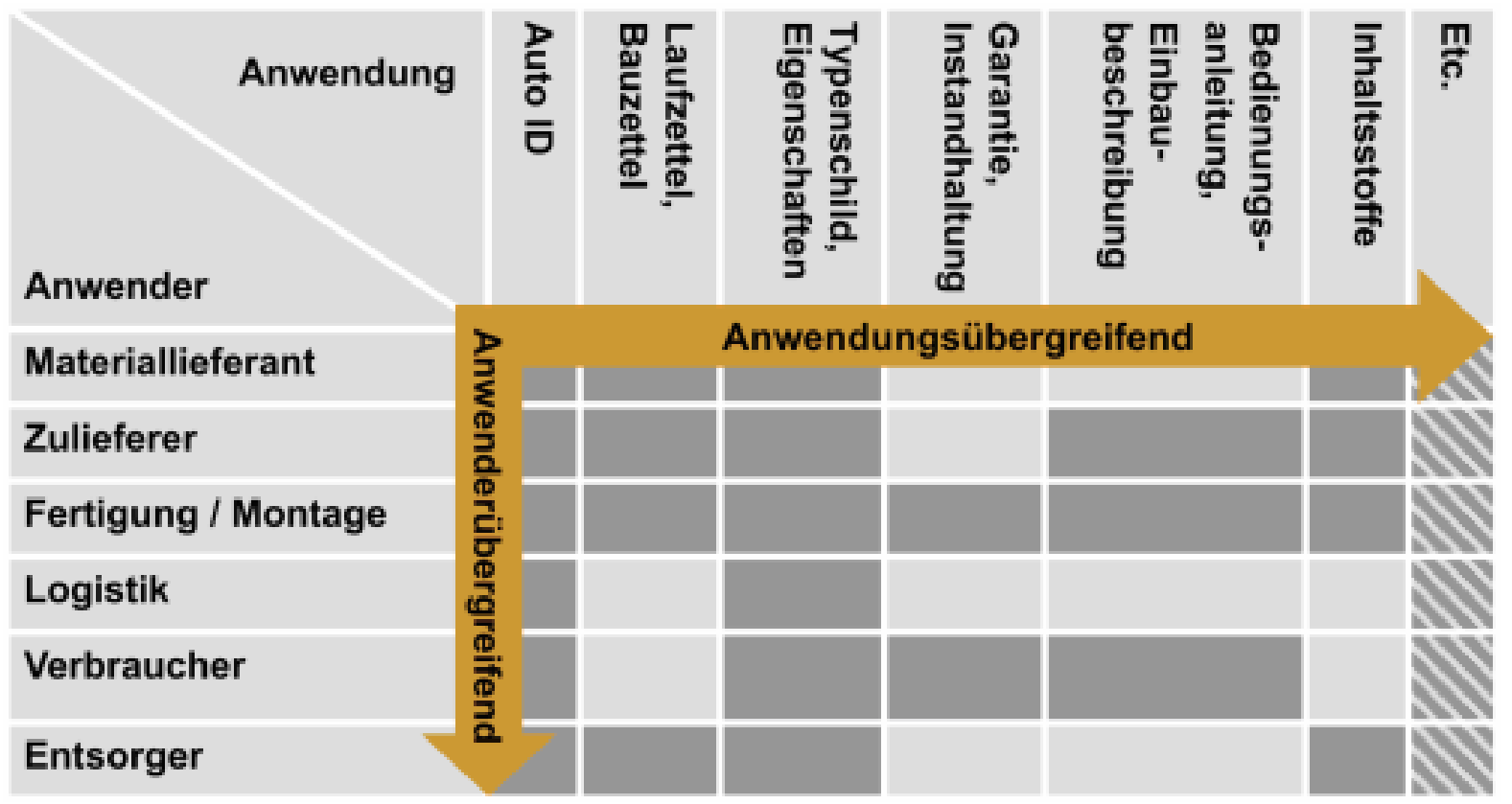

Abbildung 12: offene Systeme

\section{Literatur}

[Alles01]

[Booz04]

[DAL04]

[Dietz01]

[Finkenzeller02]

[Höhn04]

[Infinion03]

[Keller04]

[OConor04]

[Overmeyer03]

[Schröder02]
Alles, R.: Mikro-Elektronik in Gummifördergurten, Tagungsband Fachtagung, „Kautschuk und Elektronik“ der Deutschen Kautschuk - Gesellschaft (DKG), Bad Neuenahr, 26. September 2001

Booz Allen Hamilton, Universität St.Gallen: RFID-Technologie: Neuer Innovationsmotor für Logistik und Industrie?, http://www.boozallen.de/content/downloads/5h_rfid.pdf (Stand: 13.09.2004)

Delta Airlines: DAL Rolls Out Industry Leading Wireless Baggage Transfer System at Cincinnati Hub, http://news.delta.com/print_doc.cfm?article_id=9304 (Stand: 15.09.2004)

Dietz, P.: Intelligentes Transportbandsystem, Sensor Report (2001), 3. Ausgabe S. 16

Finkenzeller, Klaus: RFID-Handbuch, Carl Hanser Verlag, 2002

Höhn, R.; Overmeyer, Ludger: Logistikdatenerfassung und -verarbeitung in der Produktion auf Basis von intelligenten Transpondern, Tagungsband RFIDWorkshop an der Universität St. Gallen, 27. September 2004 Infineon Technologies, Berger, D.: Technische Grundlagen der Transpondertechnologie, Vortrag, Essen, 2003

Keller, M: Der Transponder im Fördergurt - ein Erfahrungsbericht, 9. Tagung „Zahnriemengetriebe“ am Institut Feinwerktechnik und Elektronik-Design der TU Dresden, 14./15.September 2004

O'Conor, Marie Catherine: Airline Interest in RFID on the Rise, in: RFID Journal, http://www.rfidjournal.com/article/articleprint/502/-1/1/ (Stand: 15.09.2004)

Overmeyer, Ludger: Das intelligente Transportband, Sonderheft Fördermittel Journal, Fördertechnik (2003), 15. Ausgabe S. 8-11

Schröder, Tim: Etiketten steuern Fabriken, Pictures of Future (2002), 2. Zeitschrift der Siemens AG, S. 19-22. 
[Texas04]

[VDC04]

[Ward04]

[Wennekamp04]
Texas Instruments: Blind Navigational Study at the University of Florida, in: RFID eNews, http://www.ti.com/tiris/docs/news/eNews/enewsvol34.htm, (Stand 21.09.2004)

VDC: RFID Marktdaten; in: ident (2004); 4. Ausgabe S. 9, www.vdccorp. com

Ward, Diane Marie: 5 ct tag unlikely in 4 years, www.rfidjournal.com; 30., RFID Journal, August 2004

Wennekamp, Tobias; Overmeyer, Ludger: Einsatz der RFID-Technik in Transportbändern, 9. Tagung „Zahnriemengetriebe“ am Institut Feinwerktechnik und Elektronik-Design der TU Dresden, 14./15. September 2004 